Disclosures: A. Gupta, None.

Objective: To analyze long-term functional recovery and residual deficits in patients with Guillain-Barre Syndrome (GBS).

Design: Prospective longitudinal follow-up study.

Setting: Neurological rehabilitation unit of tertiary care hospital.

Participants: Forty-one out of 330 patients (12.42\%) admitted in the institute were transferred to the neurological rehabilitation unit for inpatient rehabilitation. Twenty-six patients (M:F,14:12) reporting at 1 year follow-up (63.41\%) were included in the study (between Sept. 2005-Arpil 2008). Their functional recovery and residual deficits were recorded and analyzed.

Interventions: Not applicable.

Main Outcome Measures: Modified Barthel Index scores, Modified Rankin Scale and Hughes Disability Scale were used to assess functional disabilities at admission, discharge and follow-up.

Results: Age ranged from 4-65 years $(29.69 \pm 17.19)$. At admission, 21 patients had typical GBS and 5 had AMAN variant. Eight patients had bulbar weakness, 3 had autonomic dysfunction and 17 had facial palsy (uni/ bilateral). Nineteen patients (73.07\%) had neuropathic pain needing medication with 6 requiring more than one drug. Five patients (19.2\%) were prescribed wrist cock-up splint (WCS) for wrist drop and 15 patients (57.6\%) prescribed ankle-foot orthoses-AFO (13 bilateral AFO) for foot drop. Twenty patients $(77 \%)$ needed assistive devices also for locomotion at discharge. At 1 year follow-up, wrist drop and foot drop were still present in 1 and 4 patients, respectively, using orthoses. Modified Barthel Index scores, Modified Rankin Scale and Hughes Disability Scale were used to assess functional disabilities. Significant recovery was observed at the time of discharge and after 1 year when compared with discharge scores $(P<.001$ each).

Conclusions: GBS patients continue to show significant functional recovery for a long period. They have disability in the form of motor weakness even after 1 year and need to be followed up for longer duration.

Keywords: Functional recovery, Guillain Barre syndrome, Inpatient rehabilitation, Residual deficits.

\section{Poster 213}

\section{Health and Function Data From an Adult Cerebral Palsy Clinic: Initial Report.}

Heidi J. Haapala, MD (University of Michigan, Ann Arbor, MI); Margy A. Fox, MS; Edward A. Hurvitz, MD.

Disclosures: H. J. Haapala, None.

Objective: To describe health and functional status of adults and older adolescents with cerebral palsy enrolled in a specialty clinic.

Design: Cross sectional.

Setting: Tertiary care center.
Participants: 22 individuals (ages 17-53) with cerebral palsy, GMFCS I-V, 16 female.

Interventions: Survey, height and weight measures.

Main Outcome Measures: BMI, pain (0-10 point scale) and constipation rating scale scores, survey results, vitamin D levels.

Results: BMI: $40.9 \%$ of the participants were overweight or obese, while $31.8 \%$ were underweight. Pain: Best, worst, and average pain over the past 3 months was scored. Worst pain was rated as $\geq 7 / 10$ in $9 / 12(75 \%)$. Average pain was $0-3 / 10$ in 5 (42\%), 4-6/10 in $6(50 \%)$, and $\geq 7 / 10$ in 1 subject. Constipation: for 16 subjects, with 5 (31\%) participants scoring $>5$. Living situation: 12 lived with their parents, 3 in group homes, 1 with an aide, and 5 with roommates or by themselves. Eight of the subjects attended school, 4 worked (including individuals in sheltered living situations), and 10 neither worked nor attended school. Bone Health: As part of an initial screen, vitamin D levels were checked on 10 subjects, with below normal vitamin D levels noted in 8/10 (80\%). Mobility: 9 used manual wheelchairs, 4 used power mobility, 1 used both. GMFCS levels: 4 subjects were level 1 or 2, 10 subjects were level 3 and 8 subjects were level 4 or 5 . This group was weighted toward GMFCS 3-5, accounting for the higher prevalence of wheelchair use.

Conclusions: Vitamin D levels were low in most of the subjects, indicating a need for bone health assessment. Overweight, pain, constipation, depression, nutrition and community involvement should be monitored and addressed to increase function along with tone, mobility and other more commonly described issues in individuals with cerebral palsy.

Keywords: Cerebral palsy, Rehabilitation, Adult.

\section{Poster 214}

\section{Heroin-induced Femoral Neuropathy and Rehabilitation: A Case Report.}

Sooyeon Kim, (Montefiore Medical Center, Bronx, NY); Andrzej C. Kroszczynski, MD; Margarita Manahan.

Disclosures: S. Kim, None.

Patients or Programs: A 43-year-old male heroin abuser. Program Description: We present a 43-year-old otherwise healthy male who developed worsening bilateral lower extremities weakness and numbness for 4 months which started soon after intravenous heroin injection. Physical examination revealed marked atrophy of bilateral quadriceps, 1/5 strength of knee extensor with absent patellar reflexes, normal strength of hip flexor and adductor, along with the pattern of bilateral anteromedial thigh and medial lower leg sensory deficit consistent with bilateral femoral neuropathy or L3-4 nerve root pathology. EMG demonstrated severe bilateral femoral neuropathies with sensory and motor axonal loss. The lesions were distal to the branch supplying the iliopsoas, as this muscle was not affected. There was no evidence of lumbosacral root dysfunction. Laboratory examinations include CPK, autoimmune diseases and infectious 